\title{
Terminal sliding mode control on autonomous underwater vehicle in diving motion control
}

\author{
Nira Mawangi Sarif ${ }^{1}$, Rafidah Ngadengon ${ }^{2}$, Herdawatie Abdul Kadir ${ }^{3}$, Mohd Hafiz A. Jalil ${ }^{4}$ \\ Faculty of Electrical Engineering, Universiti Tun Hussein Onn Malaysia, Malaysia
}

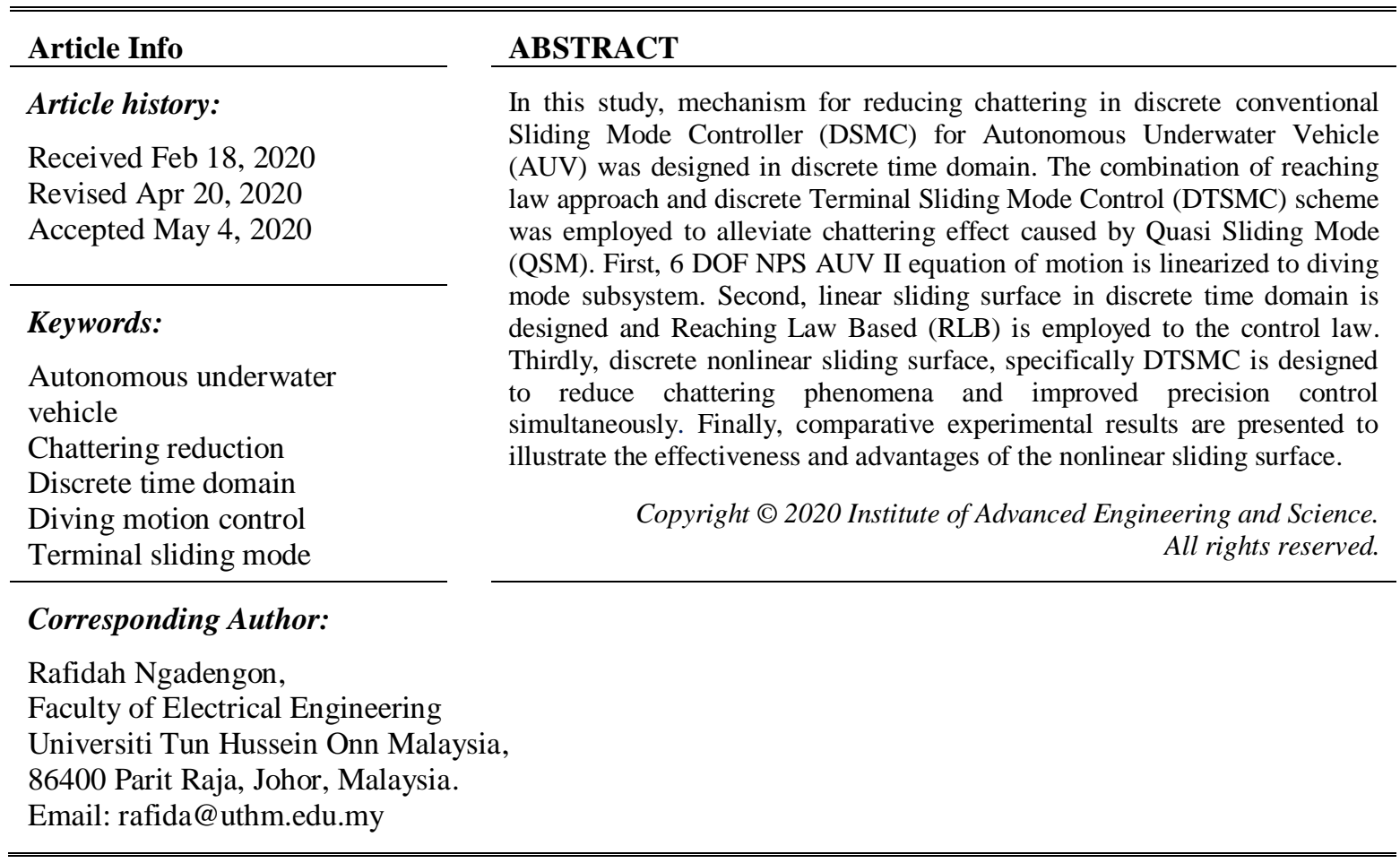

\section{INTRODUCTION}

Sliding Mode Control (SMC) is a kind of a variable structure control system introduced by Emlyanov from Soviet Union over past 50 years. Its apparent that SMC evidently a promising strategy among robust controllers [1] due to its simplicity, robustness against model uncertainties, external disturbances and parameter variations [2]. For this reason, SMC has been widely used in variety fields such as autonomous underwater vehicle [3, 4], robotics system [5, 6], power converter [7, 8] and other applications to meet motion control objectives.

Since the development in digital electronics and computer control, DSMC dramatically received a lot of attentions among researchers in recent decades [9-14]. Moreover, DSMC have advantages over SMC. Some of the advantages are less power utilization, lower cost, lighter weight, higher accuracy and easiness of masking design and software [15]. In DSMC, the control algorithm is calculated in each sampling instant and freeze as a same value until the next sampling interval. However, due to finite frequency oscillation, a zig zag motion will happen in Quasi Sliding Mode (QSM). This means the system state will move within specific band called as Quasi Sliding Mode Band (QSMB). Because of the zig zag motion in the QSM the system state cannot converge to origin in finite time. Therefore, chattering phenomena around the QSMB occurs. Chattering phenomena which refer to high frequency oscillation in the control input should be avoided because it will be degraded controller performance and decrease lifetime of the actuator and components.

Numerous strategies have been introduced in the literature to overcome the chattering problems [16-19]. One of the famous methods is employing RLB control technique where this method has been introduced and investigated in [20] and widely used until today [21-23]. The design procedure of RLB control strategy is divided into 2 steps. The first step is designing predefine profile of sliding variable so that system dynamics satisfies the desired closed system response. The second part is designing the control law such that any state outside the sliding surface is driven to reach the surface in finite time and permanently stay on the surface. 
In RLB control strategy, two of the most important properties to paid attention are chattering mitigation and convergence acceleration. There is always tradeoff between chattering mitigation in DSMC and convergence process. Specifically, chattering reduction can be achieved by choosing small control gains, however small control gains leads to slow convergence and poor robustness. Therefore, to balance tradeoff between convergence acceleration and chattering alleviation, the nonlinear sliding surface which consist of controller gain and fractional power rule employ into the controller. This new controller known as Terminal Sliding Mode Controller (TSMC). The aim of TSMC is to fasten the convergence acceleration of the system state once the sliding surface is intercepted $[24,25]$. This is to say, dynamic response of TSMC converged to origin in finite time following power rule during sliding mode stage.

Combination of both techniques TSMC and RLB strategy, is desirable to mitigate chattering effect and improve control accuracy simultaneously. Although extensive research has been conducted on DSMC, relatively limited work on DTSMC design combine with RLB strategy has been reported. To author's knowledge, no work has been reported on the design of a DTSMC combine with discrete RLB strategies dedicated to AUV applications.

The aim of this work is to design and implement DTSMC with RLB approach to alleviate the chattering effect in finite time and improved precision motion control. In this work, RLB approach with nonlinear function is design to accelerate the reaching process of the state trajectory once sliding surface reached to the origin in finite time. For this reason, the chattering effect significantly reduce, and precision control action simultaneously improved.

The value of nonlinear parameters in terminal sliding surface are gradually varying along with the varying of the terminal sliding surface $\left(S(k)_{T}\right)$. When the system state is far away from the sliding surface, the control gain is increase thus, the reaching phase will be accelerated. However, when the system state moving closed to the sliding surface, the control gain will decrease, hence the chattering effect in the input system is alleviated. Therefore, the tracking performance will be significantly improved.

The sequences of this paper are as follow: Section 2 the NPS II AUV dynamic model in depth equation of motion. Section 3, the DSMC and DTSMC is developed to the discretized model. Simulation results of NPS II AUV are given in Section 4 and 5 respectively.

\section{MODELLING AUTONOMOUS UNDERWATER VEHICLE}

A complete dynamic of AUV motion has 6 degree of freedom (DOF) rigid body in fluid dynamic are described by a set of 12 nonlinear, coupled, first-order differential equations with constant coefficients [26]. In order to reduce the complexity in designing control law, this scope restricts to dive motion with horizontal motion control parameters are set at zero. Next, pitch and heave equation of motion of the vehicle in the body coordinate fixed frame are given by

$$
\begin{aligned}
& m\left[\dot{w}-u_{0} q\right]=Z \\
& I_{y} \dot{q}=M \\
& Z=Z_{\dot{w}} \dot{w}+Z_{\dot{q}} \dot{q}+Z_{w} w+Z_{q} q+Z_{\delta} \delta_{s} \\
& M=M_{\dot{w}} \dot{w}+M_{\dot{q}} \dot{q}+M_{w} w+M_{q} q-m g\left(Z_{G}-Z_{B}\right)+M_{\delta} \delta_{s}
\end{aligned}
$$

Where $u_{o}$ is vehicle's designed velocity, $w$ is heave velocity, $\theta$ is pitch angle, $q$ is pitch angle velocity, $z$ is vehicle depth, $\delta_{s}$ is control fin angle, $F_{p}$ is the propulsion force control forward velocity, $m$ is vehicles mass, $I_{y y}$ is moment of inertia of the vehicle about the pitch axis, $W$ denotes the vehicle's weight and $z_{G}$ is vehicle centre gravity, $Z_{B}$ is vehicle centre of buoyance. The hydrodynamic parameters [27] are included added mass: $Z_{\dot{q}}, Z_{\dot{w}}, M_{\dot{q}}, \quad M_{\dot{w}}$, cross flow drag: $Z_{q}, Z_{w}, M_{q}, M_{w}$ and finally fin list moment and force: $M_{\delta}$ and $Z_{\delta}$.

Defining the state vector $x=\left[\begin{array}{llll}x_{1} & x_{2} & x_{3} & x_{4}\end{array}\right]^{T}=\left[\begin{array}{lll}w & q \theta & z\end{array}\right]$, the control vector $v=\left[\delta_{s}\right]$, the linearized diving equation of motion matrix written in the following form.

$$
\left[\begin{array}{cccc}
m-Z_{\dot{w}} & -Z_{\dot{q}} & 0 & 0 \\
-M_{\dot{w}} & I_{y}-M_{\dot{q}} & 0 & 0 \\
0 & 0 & 1 & 0 \\
0 & 0 & 0 & 1
\end{array}\right]\left[\begin{array}{c}
\dot{w} \\
\dot{q} \\
\dot{\theta} \\
\dot{z}
\end{array}\right]+\left[\begin{array}{cccc}
-Z_{w} & m u_{0}-Z_{q} & 0 & 0 \\
-M_{w} & -M_{q} & \left(Z_{G}-Z_{B}\right) W & 0 \\
0 & -1 & 0 & 0 \\
-1 & 0 & u_{0} & 0
\end{array}\right]\left[\begin{array}{c}
W \\
q \\
\theta \\
z
\end{array}\right]=\left[\begin{array}{c}
Z_{\delta} \\
M_{\delta} \\
\theta \\
z
\end{array}\right] \delta_{s}
$$


As in [28], the heave velocity during diving is very small (less than $0.05 \mathrm{~m} / \mathrm{s}$ ), thus term containing $w$ and $\dot{w}$ can be neglected. Since $w$ and $\dot{w}$ are neglected, the mathematical model of diving control as in as shown in (5) illustrated in state space form as:

$$
\left[\begin{array}{c}
\dot{q} \\
\dot{\theta} \\
\dot{Z}
\end{array}\right]=\left[\begin{array}{ccc}
\frac{M_{q}}{I_{y}-M_{\dot{q}}} & \frac{-W\left(Z_{G}-Z_{B}\right)}{I_{y}-M_{\dot{q}}} & 0 \\
1 & 0 & 0 \\
0 & -u_{0} & 0
\end{array}\right]\left[\begin{array}{c}
q \\
\theta \\
Z
\end{array}\right]+\left[\begin{array}{c}
\frac{M_{\delta}}{I_{y}-M_{\dot{q}}} \\
0 \\
0
\end{array}\right] \delta_{s}
$$

The vehicle dynamic (2) are represents in the typical representation of linear systems:

$$
\begin{aligned}
& \dot{x}(t)=A x(t)+B u(t) \\
& y(t)=C x(t)
\end{aligned}
$$

\section{CONTROLLER DESIGN}

\subsection{Discrete-time linear sliding surface (LSMC)} described as:

Consider a continuous time system in (7), the discrete model of (1) using Euler approximation

$$
\begin{aligned}
& x(k+1)=\Phi x(k)+\Gamma u(k) \\
& y(k)=H x(k)
\end{aligned}
$$

where $x(k)$ is the state vector, $u(k)$ is the control input and $y(k)$ is the output and $\Phi$ and $\Gamma$ is the system matrices.

The control objective is to force the variable $x$ to achieve a constant reference position $x_{r}$. Let's define the tracking error as:

$$
e=x_{r}-x
$$

Then the discrete conventional sliding surface is stated as follows:

$$
\begin{aligned}
& S(k)=C_{s} e(k) \\
& =C_{s}\left(x_{r}(k)-x(k)\right)
\end{aligned}
$$

Where $e(k)$ is the tracking error, $x_{r}$ is reference input and $C_{s}$ is the sliding matrix selected such that $C_{s}$ is gain matrix.

The system is designed to steer the state trajectory to the origin when travelling along switching surface. Without loss of generality, the following linear sliding surface defined as:

$$
S(k)=0
$$

Considering as shown in (10), the first-time derivative of the sliding surface is given by:

$$
S(k+1)-s(k)=0
$$

Using this condition, the RLB method described by [20] defined as;

$$
\begin{aligned}
& S(k+1)-S(k)=-q T S(k)-\varepsilon \operatorname{Tsgn}(S(k)) \\
& S(k+1)=(1-q t) S(k)-\varepsilon \operatorname{Tsgn}(S(k))
\end{aligned}
$$

Where $T$ is the sampling interval of discrete time system, $\varepsilon$ and $q$ are positives constants. $\varepsilon>0$,

$$
q>0 \text { and } 1-q T>0 \text {. }
$$

From (10), (12) and reaching law in (13), one step forward of the sliding surface is given by: 


$$
S(k+1)-S(k)=\left[\begin{array}{c}
C_{s} x_{r}(k+1)-C_{s} \Phi x(k)-C_{s} \Gamma u(k)-C_{s} x_{r}(k)+C_{s} x(k) \\
-q T S(k)-\varepsilon T s g n(S(k))
\end{array}\right]
$$

Employing reaching law in (13) - (14), therefore the DSMC control law for system (8) expressed as:

$$
u(k)=-\left(C_{s} \Gamma\right)^{-1}\left[-C_{s} x_{r}(k+1)+C_{s} \Phi x(k)+(1-q T) s(k)-\varepsilon T \operatorname{sgn}(s(k))\right]
$$

\subsection{Discrete-time nonlinear sliding surface (TSMC)}

The nonlinear terminal sliding surface in discrete-time domain is expressed as:

$$
S_{T}(k)=C_{T} e(k)+\beta e_{1}^{\frac{q}{p}}(k-1)
$$

Where $\beta>0, p$ and $q$ are both positive odd integers such that $q>p, 0<\frac{q}{p}<1$ and $C_{T}$ is terminal sliding gain.

The sliding mode is obtained if the following condition as verified:

$$
S_{T}(k)=S_{T}(k+1)=0
$$

Using condition in (17) and reaching law described in (13), forward expression of sliding mode (17) expended as:

$$
S_{T}(k+1)-S_{T}(k)=\left[\begin{array}{c}
C_{T} x_{r}(k+1)-C_{T} \Phi x(k)-C_{T} \Gamma u_{T}(k)+\beta x_{1}{ }^{\frac{q}{p}} S(k) \\
-q \operatorname{tS}(k)-\operatorname{cTsgn}(S(k)
\end{array}\right]
$$

So, the terminal control law-based reaching law approach can be express as:

$$
u_{T}(k)=-\left(C_{T} \Gamma\right)^{-1}\left[\begin{array}{c}
-C_{T} x_{r}(k+1)+C_{T} \Phi x(k)-\beta x_{1}{ }^{\frac{q}{p}}(k) \\
+(1-q T) s(k)-\operatorname{\varepsilon Tsgn}(s(k))
\end{array}\right]
$$

\section{SIMULATION STUDIES}

DTSMC and DSMC control scheme are applied to the model of NPS AUV II during vertical plane is described by dynamic equations of motion in (6). Computer simulations are performed considering the NPS AUV II model parameter as presented in Table 1. In this simulations, initial state is taken to be zero (i.e, the vehicle is at rest) such that $x(0)=y(0)=z(0)=q(0)=\theta(0)=0$. The step input is set after $5 \mathrm{~s}$. The nonlinear component added into sliding function determined in (16) to reduce chattering effect and improve precision control simultaneously. To show the effectiveness of the proposed controller, both conventional discrete linear SMC(LSMC) (15) and discrete nonlinear SMC (TSMC) schemes (19) are investigated for comparisons. The parameters of both controllers are carefully selected as shown in Table 2. The parameters have verified to obtained satisfactory performance without considering external uncertainty.

The output position, pitch angle and control input are displayed in Figure 1, Figure 2 and Figure 3. It can be noticed, both controllers achieved good results. The main reason is that, both controllers employ with very famous Gao's reaching law as in as shown in (13). This is because, the nonlinear component in TSMC sliding surface (16) forced the system to converge to origin during sliding mode. Although, TSMC doesn't not provide a fast convergence over time, the undesirable dynamic behaviour called as chattering phenomena in LSMC signifyingly alleviated in TSMC as illustrated in Figure 4.

It is notable that $q / p=1$ and $\beta=0$ lead to linear sliding surface. Therefore, by tuning these two parameters while keeping the other parameter shown in Table 1 different controllers obtained. The selection of control parameter $\beta$ and $q / p$ are chosen according to error bound and convergence time specification of position tracking error respectively. The influences of different $\beta$ and ${ }^{q} / p$ values are shown in Figure 5 and Figure 6. In this case, the convergence time of position tracking error is around 500sec where bigger value of $\beta$ and $q / p$ provide smaller error bound and faster error convergence respectively. It should be noted that, the selection of $\beta$ and $q / p$ is within the range of the stability condition. Therefore, proper selection of TSMC parameters improved precision control as illustrated in Figure 7. Discrete nonlinear sliding surface (DTSMC), provide better position tracking control and better dynamic response. 
Table 1. The NPS AUV II model parameter

\begin{tabular}{ccc}
\hline Parameter & Value & Units \\
\hline$m$ & 5443.4 & $\mathrm{Kg}$ \\
$W$ & 53400 & $\mathrm{~N}$ \\
$Z_{G}$ & 0.061 & $\mathrm{M}$ \\
$Z_{B}$ & 0 & $\mathrm{~m}$ \\
$I_{y}$ & 13587 & $\mathrm{Nms}^{2}$ \\
$M_{\dot{q}}$ & $-1.7 \times 10^{-2}$ & - \\
\hline
\end{tabular}

Table 2. Controller parameters

\begin{tabular}{ll}
\hline \multicolumn{1}{c}{ Control Algorithms } & Controller Parameter \\
\hline DTSMC & $t=0.2 \mathrm{sec}, q=1, \varepsilon=0.001, \beta=0.0003,{ }^{q} / p=1 / 5$ \\
DSMC & $t=0.2 \mathrm{sec}, q=1, \varepsilon=0.001$ \\
\hline
\end{tabular}

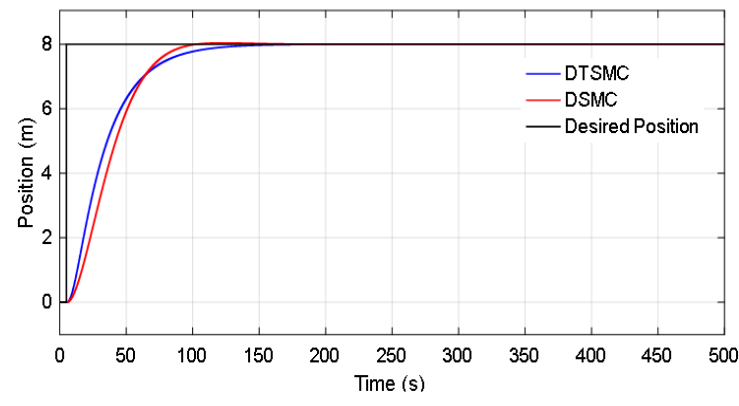

Figure 1. Vehicle depth respond

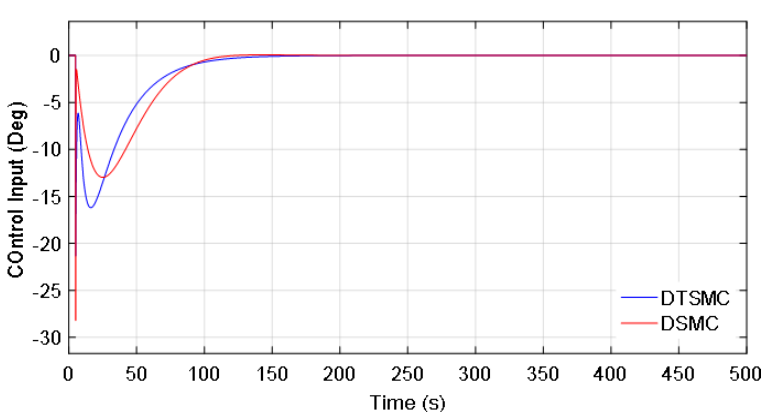

Figure 3. Control input evolution

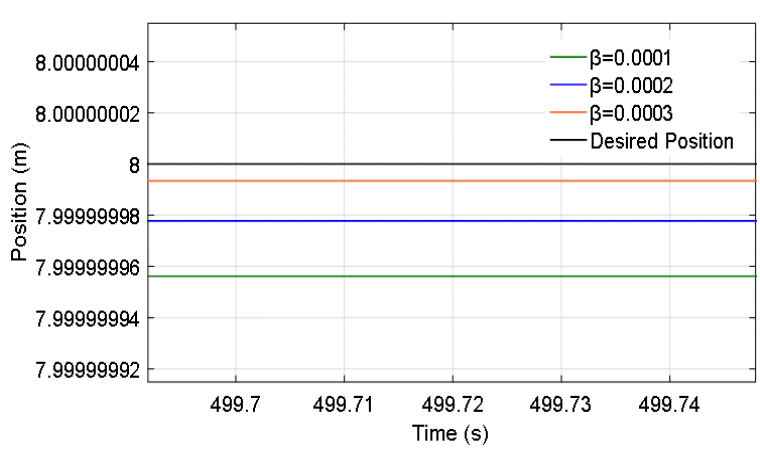

Figure 5. Error displacement for different value of

$$
\beta \text { and } q / p=5 / 9
$$

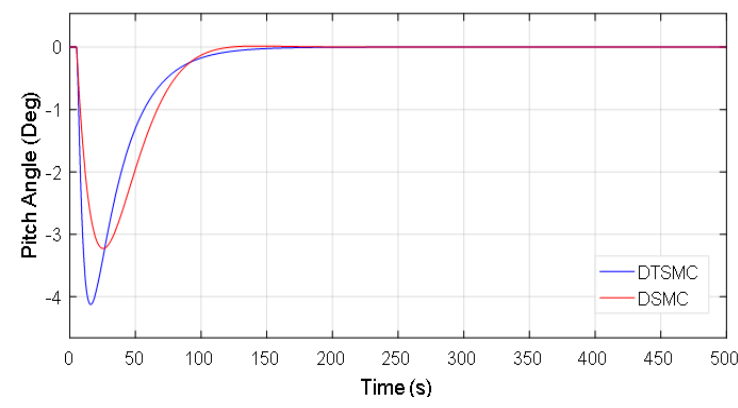

Figure 2. Vehicle pitch angle respond

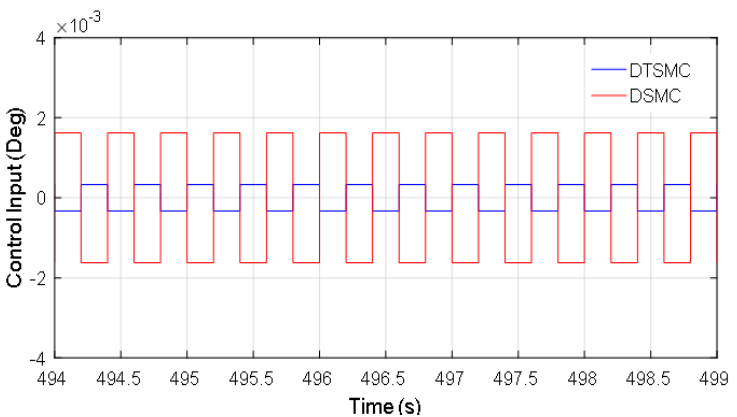

Figure 4. Chattering effect in control Input

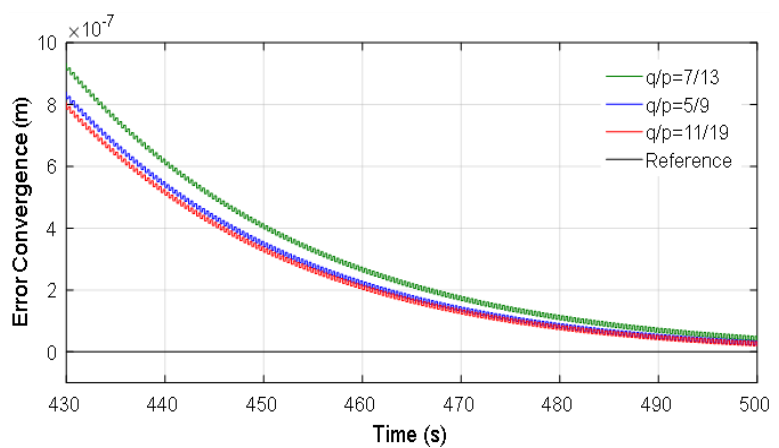

Figure 6. Error convergence for different pair of $q / p$ and $\beta=0.0003$ 


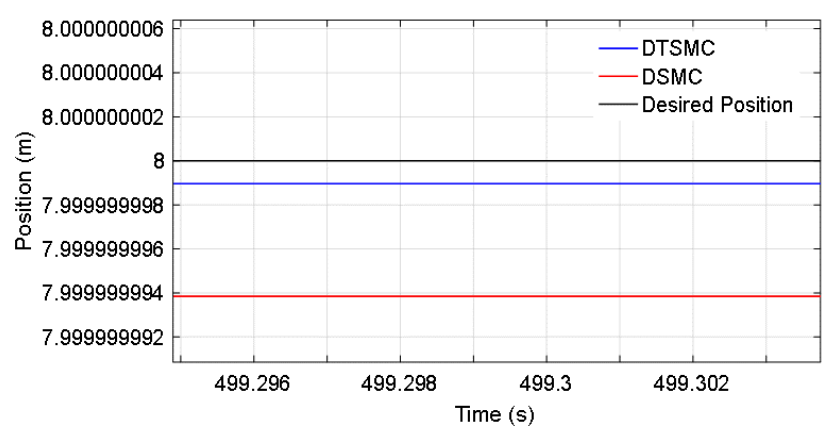

Figure 7. Precision control of DTSMC and DSMC

\section{CONCLUSION}

In this study, the issue on chattering phenomena of internal dynamics system existed on DSMC has been investigated. To mitigate the chattering phenomena caused by QSM, firstly a discrete nonlinear sliding surface based on DTSMC method is introduced. Because of the nonlinear function involved in the reaching law, the system states will converge to the origin once the sliding surface intercepted in finite sample steps. As a result, the chattering effect significantly alleviated. However, some limitations still exist on proposed controller where the trajectory convergence slower than DSMC when the system state far away from the origin. Therefore, this research will concentrate on how to improve the trajectory convergence although the system state far away from the origin. Secondly, the error bound and convergence rate is analysed by varying both control parameters of the nonlinear component in TSMC. Thirdly, the tracking state accuracy is significantly improved. Finally, comparison examples are carried out to illustrate the advantages of combination DTSMC and RLB strategy.

\section{ACKNOWLEDGEMENTS}

The authors would like to thank Universiti Tun Hussein Onn Malaysia (UTHM) for TIER 1 grant Vot H148,GPPS grant Vot H316 and AdMiRe FKEE for the research funding support.

\section{REFERENCES}

[1] V. I. Utkin, "Variable Structure Systems with Sliding Modes," IEEE Trans. Automat. Contr., vol. 22, no. 2, pp. 212-222, 1967.

[2] K. D. Young, V. I. Utkin, and Ü. Özgüner, "A control engineer's guide to sliding mode control," IEEE Trans. Control Syst. Technol., vol. 7, no. 3, pp. 328-342, 1999.

[3] M. R. Ramezani-al and Z. Tavanaei-Sereshki, "An adaptive sliding mode controller with a new reaching law for tracking problem of an autonomous underwater vehicles," Trans. Inst. Meas. Control, vol. 41, no. 6, pp. 1772-1787, 2019.

[4] A. N and D. V. D. Shahab Heshmati-alamdari, "Robust Trajectory Tracking Control for Underactuated Autonomous Underwater Vehicles," in IEEE 58th Conference on Decision and Control (CDC), 2019.

[5] L. Xi, S. Yang, H. Dong, and X. Qi, "Terminal Sliding Mode Control for Robotic Manipulator Based on Combined Reaching Law," Proc. - 2nd Int. Conf. Comput. Network, Electron. Autom. ICCNEA 2019, pp. 436-441, 2019.

[6] S. Islam and X. P. Liu, "Robust sliding mode control for robot manipulators," IEEE Trans. Ind. Electron., vol. 58, no. 6, pp. 2444-2453, 2011.

[7] Y. Liu et al., "DFIG wind turbine sliding mode control with exponential reaching law under variable wind speed," Int. J. Electr. Power Energy Syst., vol. 96, pp. 253-260, 2018.

[8] B. Yang, T. Yu, H. Shu, J. Dong, and L. Jiang, "Robust sliding-mode control of wind energy conversion systems for optimal power extraction via nonlinear perturbation observers," Appl. Energy, vol. 210, pp. 711-723, 2018.

[9] B. Wang, "On Discretization of Sliding Mode Control Systems On Discretization of Sliding Mode Control Systems (Phd Thesis)," RMIT University Melbourne, Australia, 2008.

[10] K. Furuta, "Sliding mode control of a discrete system," Syst. Control Lett., vol. 14, no. 2, pp. 145-152, Feb 1990.

[11] A. Bartoszewicz, "Discrete-Time Quasi-Sliding-Mode Control Strategies," in Industrial Electronics, IEEE Transactions on, vol. 45, no. 4, pp. 633-637, 1998.

[12] G. Monsees, Discrete-Time Sliding Mode Control, Technical University Delft, 2002.

[13] S. Janardhanan and B. Bandyopadhyay, "On discretization of continuous-time terminal sliding mode," IEEE Trans. Automat. Contr., vol. 51, no. 9, pp. 1532-1536, 2006.

[14] S. K. Spurgeon, "Hyperplane design techniques for discrete-time variable structure control systems," Int. J. Control, vol. 55, no. 2, pp. 445-456, 1992. 
[15] S. S. Majidabad and H. T. Shandiz, "Discrete-time based sliding-mode control of robot manipulators," International Journal of Intelligent Computing and Cybernetics, vol. 5, no. 3, pp. 340-358, 2012.

[16] B. Veselić, B. Peruničić-Draženović, and Č. Milosavljević, "Improved discrete-time sliding-mode position control using Euler velocity estimation," IEEE Trans. Ind. Electron., vol. 57, no. 11, pp. 3840-3847, 2010.

[17] V. Acary, B. Brogliato, and Y. V. Orlov, "Comments on "Chattering-Free Digital Sliding-Mode Control with State Observer and Disturbance Rejection," IEEE Trans. Automat. Contr., vol. 61, no. 11, pp. 3707, 2016.

[18] B. Wang, B. Brogliato, V. Acary, A. Boubakir, and F. Plestan, "Experimental Comparisons between Implicit and Explicit Implementations of Discrete-Time Sliding Mode Controllers: Toward Input and Output Chattering Suppression," IEEE Trans. Control Syst. Technol., vol. 23, no. 5, pp. 1-6, 2015.

[19] H. Du, X. Yu, M. Z. Q. Chen, and S. Li, Chattering-free discrete-time sliding mode control, Automatica, 2016.

[20] W. Gao, Y. Wang, and A. Homaifa, "Discrete-Time Variable Structure Control Systems," IEEE Trans. Ind. Electron., vol. 42, no. 2, pp. 117-122, 1995.

[21] H. Ma, J. Wu, and Z. Xiong, "Discrete-Time Sliding-Mode Control with Improved Quasi-Sliding-Mode Domain," IEEE Trans. Ind. Electron., vol. 63, no. 10, pp. 6292-6304, 2016.

[22] A. Bartoszewicz and P. Latosiński, "Reaching law for DSMC systems with relative degree 2 switching variable," Int. J. Control, vol. 90, no. 8, pp. 1626-1638, 2017.

[23] S. Chakrabarty and A. Bartoszewicz, "Improved robustness and performance of discrete time sliding mode control systems," ISA Trans., vol. 65, pp. 143-149, 2016.

[24] Y. Feng, X. Yu, and Z. Man, "Non-singular terminal sliding mode control of rigid manipulators," Automatica, vol. 38, no. 12, pp. 2159-2167, 2002.

[25] Y. Feng, F. Han, and X. Yu, "Chattering free full-order sliding-mode control," Automatica, vol. 50, no. 4, pp. 1310-1314, 2014.

[26] Gertler and Hagen, Standard Equations of motion for submarine simulations, Nav. Sh. Res. Dev. Cent., 1967.

[27] David C. Warne, Design, Simulation and Experimental Verification Of A Computer Model And Enhanced Position Estimator For The NPS AUV II, Naval Postgraduate School,Montery California, 1991.

[28] B. Jalving, "The NDRE-AUV Flight Control System," IEEE Journal of Oceanic Engineering, vol. 19, no. 4. pp. 497-501, 1994. 